\title{
Foggy Day Image Sharpening Algorithm Based on Depth of Field Estimation
}

\author{
Changli Li and Qian Jia \\ Advanced Signal and IMage Processing, Learning and Engineering Lab (A Simple Lab), College of Computer and Information, \\ Hohai University, Nanjing 211100, China
}

\begin{abstract}
Traditional dehazing methods always use the dark channel a priori method to recover the original image, which leads to the calculation of the transmission is large and takes too much time. Therefore, in this paper, we present a fog-day-based image sharpening algorithm based on depth of field estimation. This approach makes use of white balancing, which eliminates the color cast that is caused by the atmospheric color. Then we extract the depth features of the haze image and construct the depth of field estimation model. Finally, the original image is restored by estimating the depth of field of each pixel of the image. The experimental results show that the method we de veloped has the capability to remove the haze efficiently, by which the finest details and edges are enhanced significantly.
\end{abstract}

Keywords- Image dehazing; depth of field estimation; White balancing; feature extraction

\section{INTRODUCTION}

Currently there are two main types of dehazing methods available: image enhancement algorithm and physical model-based methods. The traditional image enhancement methods are based on the histogram equalization algorith $\mathrm{m} \mathrm{[1],}$ based on the Retinex model algorithm [2] and wavelet transform based enhancement [3]. Although these methods can enhance the image, improve the quality of the image and apply to the existing mature image processing algorithms. However, due to ignoring the misty image degradation mechanism, it may cause some loss of image information and image distortion. Demystification algorithms based on physical models have made significant progress, using atmospheric scattering models to restore foggy images to fog-free images, where depth-based methods obtain depth information in the image using some means and then solve The parameters of the image degradation model are extracted and the parameters are substituted into the model to retrieve the foggy image. The dark channel theory proposed by He et al. [4] can get better reconstructed images when dealing with haze images, but the calculation of the optimal transmission by the matting algorith $m$ is very large and takes a long time; the sky and other colors are gray and white of the bright areas produce color distortion, dark channel theory does not hold in this environment. In order to overcome the weakness es of the dark channel theory, scholars have tried to adopt much improvement.

Zhu et al. [5] proposes a priori algorith $\mathrm{m}$ based on color attenuation under the condition of unknown scene depth of field. The concentration of fog is estimated by extracting the difference between saturation and brightness information of the image scene. According to the concentration of fog, the scene depth of field depth is estimated and then the image is restored. Although the color and the visual experience of the processed image are greatly improved, the defogging effect is not stable. When dealing with the images of some special scenes, the defogging force is obviously not enough.

Therefore, aiming at the problems existing in the above algorithms, this paper proposes a foggy image sharpening algorith $m$ based on depth of field estimation. Firstly, we adopt the white balance correction and get the color correction chart, restore the original color of the fog. Secondly, extract the brightness and texture feature information by the haze image which can effectively reflect the current depth of field of each pixel. Then, the depth of field estimation model is built to estimate the depth of field of each pixel of the image, which is substituted into the atmospheric scattering model to recover the original image. Finally, the leading filter is used to improve the edge structure of the depth image, hence the reconstructed image will probably become more smooth.

\section{FogGY IMAGE FogGING METHOD BASED ON DEPTH OF FIELD ESTIMATION}

\section{(1) Atmospheric scattering model:}

In the field of computer vision and image processing, the physical model of atmospheric degradation widely used in foggy image processing is [6]:

$$
I(x)=J(x) e^{-\beta d(x)}+A\left(1-e^{-\beta d(x)}\right)
$$

where $I(x)$ is achieved image, $J(x)$ is the scene radiance, $x$ is a pixel, $A$ is the atmospheric light, $\beta$ is the atmospheric particle scattering coefficient, $d$ is the scene depth of field, $J(x) e^{-\beta d(x)}$ is the direct attenuation of the target radiation information, $A\left(1-e^{-\beta d(x)}\right)$ represents the atmospheric light attenuated and reaches the imaging device energy.

\section{(2) White balance correction:}

$\mathrm{He}$ finds the maximum value of the pixel in the corresponding position by setting $0.1 \%$ of the pixels in the dark channel value and sets it as the atmospheric light value [7]. Although this estimation method is more accurate, due to the special circumstances such as white objects and strong light in the scene, atmospheric light value estimation is too 
high. Therefore, the restored image is prone to color cast. It also affects the transmittance estimation and high pixel value doesn't reflect the real atmospheric light. In order to restore the fog of the original color, this paper will firstly accomplish white balance correction from the foggy image.

The white point (WP) algorithm, also known as the Max-RGB algorith $m$ [8], uses the maximu $m$ value of the $R, G$ and B color components to estimate the color of the light. White balance the atmospheric light, the image is divided by the atmospheric light value, the formula (1) can be rewritten as:

$$
\frac{I(x)}{A}=\frac{J(x) e^{-\beta d(x)}}{A}+\left(1-e^{-\beta d(x)}\right)
$$

where $I(x) / A>1$. In order to estimate the depth map in the next section, we limit the white-balance corrected image $I(x) / A$ to the interval $[0,1]$ and use $I^{\prime}(x)$ to represent the atmospheric scattering model as:

$$
I^{\prime}(x)=\min (I(x) / A)
$$

Here the atmospheric light $A$ is corrected to white $(1,1,1)^{T}$.

\section{(3) Deep clues extraction:}

Under normal circumstances, the larger the proportion of the atmospheric light in the imaging, the higher brightness and blurred textures there is in the area [9-10]. Because of the brightness value of each pixel in the foggy image, it increases as the fog concentration increases. We transform the foggy image from RGB space to HSL space and get the luminance feature information of haze image $D_{L}$. Texture features of objects, especially of those with a regular shape or lighter colors are very important for estimating the depth of scene. In particular, it is helpful to judge the depth of scene in outdoor scenes. The texture information of any haze image is extracted by:

$$
\mathrm{D}_{\mathrm{F}}(x)=\frac{1}{|\Omega|} \bullet \sum_{x \in \Omega(x)}|\nabla I(x)|
$$

where $\Omega(\mathrm{x})$ denotes the neighborhood centered at the pixel $x$ and $|\Omega|$ denotes the number of pixels in the neighborhood, $\nabla I$ is the texture map of the foggy image.

(4) The scene radiance recovery:

With the depth map is obtained by estimating the scene depth $d$ by the brightness features $D_{L}$ and the texture features of the pixels $D_{L}$ in the extracted image:

$$
d_{r}(x)=\min _{y \in \Omega_{r}(x)} d(x)
$$

where $\Omega_{r}(x)$ is the neighborhood of the pixel $x, d_{r}$ is the depth map of the area.

When the global atmos pheric light and the scene depth of field in the atmospheric scattering model are known, the expression of the restored image can be obtained:

$$
J(x)=\frac{I(x)-A}{e^{-\beta d(x)}}+A
$$

\section{PROPOSED METHOD}

Compared with mentioned algorithms, the proposed algorith $\mathrm{m}$ has the following advantages: (1) Our algorith $\mathrm{m}$ can extract the current depth of field brightness and texture feature information effectively, which overcome the limitations of the most algorithm, which relies solely on color features to estimate the scene depth; (2) The Proposed method calculates the white balancing of the input image, which discards the color shifting obtains the color correction map, which made the color of fog is in line with human visual experience; (3) The proposed algorithm overcomes the problem of time-consuming and computationally expensive for the traditional method based on dark channel; (4) The details and edges will be enhanced by using the guided filter.

The algorithm's workflow is shown in Figure 1. The proposed algorithm extracts the brightness features $D_{L}$ and the texture features $D_{F}$ in the image, then estimates the relation between the characteristic information of the image and the concentration function of the fog $W(x)$ :

$$
W(x)=\lambda_{0}+\lambda_{1} D_{L}-\lambda_{2} D_{F}
$$

Since the depth of scene depth and the fog concentration function $W(x)$ is positively related to the relationship, so the depth of the scene depth of the image is expressed as:

$$
d(x) \propto W(x)=\lambda_{0}+\lambda_{1} D_{L}-\lambda_{2} D_{F}
$$

where the larger the weight coefficient $\lambda_{2}$, the higher the influence of the texture features on the fog concentration, and vice versa, the fog concentration function $W(x)$, the scene depth estimation model of each pixel in the picture can be rewritten as:

$$
d(x)=Q(x) W(x)+\varepsilon(x)
$$




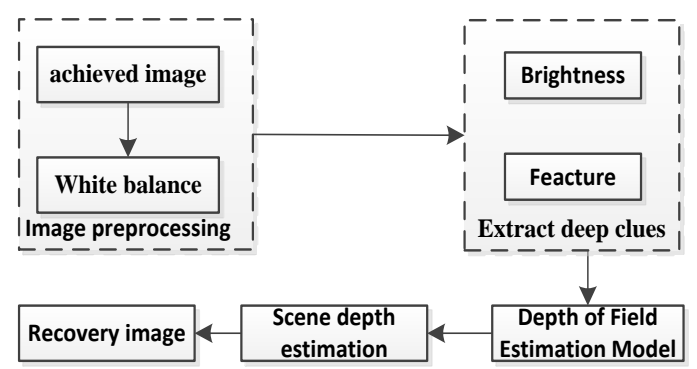

FIGURE I. ALGORITHM FRAME DIAGRAM

where $\varepsilon(x)$ is the random error variable, $Q(x)$ is the transcendental trade-off coefficient, which represents the depth contribution of the first priori graph $i_{t h}$, since we pick all the same hypotheses $Q(x)$ from the same color assumptions and pick the same $d(x)$, and $Q(x)$ satisfies that $\sum Q(x)=1$. The model can be simplified as:

$$
d(x)=W(x)+\varepsilon(x)
$$

where $\varepsilon(x)$ is a Gaussian variable $\varepsilon(x) \sim N\left(0, \sigma^{2}\right)$ with zero mean and a variance of $\sigma^{2}$. Considering the possibility of a priori uncertainty, we have:

$$
d(x)=N\left(d \mid W, \sigma^{2}\right) \propto p\left(d(x) \mid x, \lambda_{0}, \lambda_{1}, \lambda_{2}, \sigma^{2}\right)(11)
$$

Through repeated tests, we can get a more accurate scene depth depth estimation with the parameters $\lambda_{0}=0.122$, $\lambda_{1}=0.958, \lambda_{2}=0.776$.

Finally, we use the guided image filter [13] to preserve the original depth information as much as possible to eliminate the texture details, optimize the depth map obtained to enhance its edge and the fine details. So the recovery image $d_{\text {refine }}$ can be optimized:

$$
d_{\text {refine }}(x)=\sum_{y} W_{x y}(I, \varepsilon, r)(d(y))
$$

where $W_{x y}(I, \varepsilon, r)$ corresponds to the weight assigned to a pixel $y$ inside a local region centered at pixel $x$.

In order to avoid creating too much noise, we restrict the transmission to a lower bound, which varies between 0.1 and 0.9 , and the finally scene radiance is written as

$$
J(x)=\frac{I(x)-A}{\min \left\{\max \left\{e^{-\beta d_{\text {name }}(x)}, 0.1\right\}, 0.9\right\}}+A
$$

Typically, we choose $\beta=1.0$ in practice, the algorithm is better able to handle different types of foggy images as shown in Figure 2:

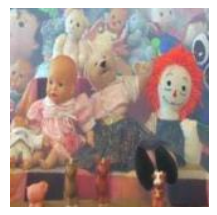

(a) Original

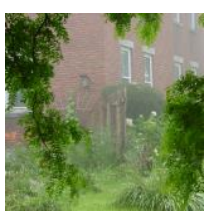

(d) Original

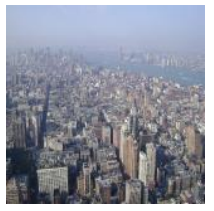

(g) Original

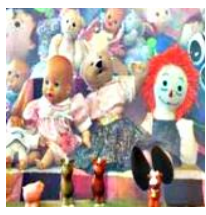

(b) Restored

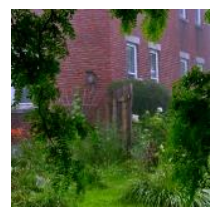

(e) Restored

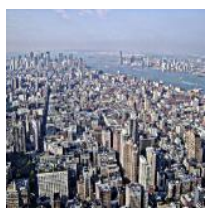

(h) Restored

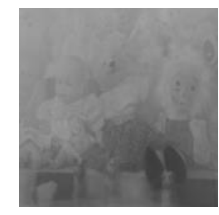

(c) Scene depth map

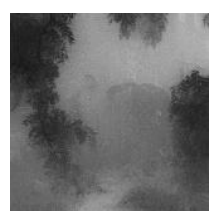

(f) Scene depth map

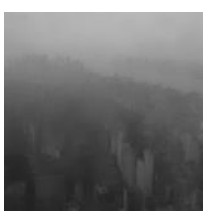

(i) Scene depth map
FIGURE II. ALGORITHM FOG EFFECT (LEFT: FOGGY IMAGE; MEDIUM: RECONSTRUCTED IMAGE; RIGHT: SCENE DEPTH MAP)

\section{EXPERIMENT RESULTS}

\section{(1) Subjective comparison}

We select different types of challenging typical scene graphs using simulations, such as close-up images, long-term images and sky images. Figure 2 shows the results and Figure 3 shows the comparis on of the proposed method with currently used stated-of-art methods.

Zhu algorithm [5], Tarel algorith $m$ [11], Retine $x$ algorith $m$ [12], He algorithm [4] and the effect of the algorithm in this paper. It can be seen from the experiment results shown in Figure 3 that the de-fogging effect of Zhu algorithm is not stable and the de-fogging power is obviously not enough. Tarel's algorith $m$ can highlight the texture details of the target scene, however, with obvious over-enhancement in visual effects, A small nu mber of Halo effects appear in the group (3) of FIGURE 3 when the algorithm is used to deal with the drastic depth change. The Retinex algorithm, although able to highlight a large number of texture details, appears to be over saturated. Good, can effectively improve the subjective visual quality of the image, but often distortions exist when dealing with the target scene with atmospheric light; In contrast, the algorith $m$ to the fog more thoroughly, the color reproduction is also higher, the recovery of the image in Visually more pleasing. In addition, in the experiment of group (3) in FIGURE 3, the proposed algorithm has almost no distortion and the processed sky is very close to the real scene, while other algorithms have been damaged in varying degrees, which further illustrates that our algorithm in this paper has a strong adaptability.

(2) Objective comparison

The complexity of the algorithm plays a crucial role in the 
real-time visual system. The real-time performance of each algorithm is shown in Table 1. Table 1 shows that our algorithm can speed up the computation.

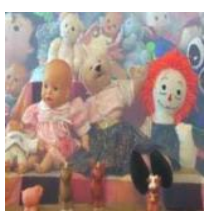

(a)Original

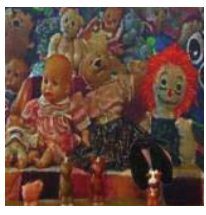

(d) Tarel's

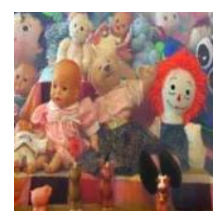

(b) He's

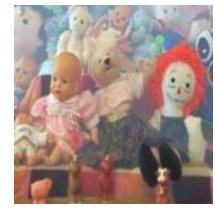

(e) Zhu's

Group I-Doll

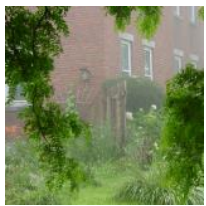

(a)Original

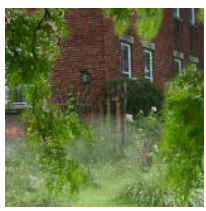

(d) Tarel's

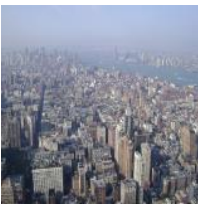

(a)Original

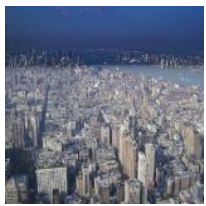

(d) Tarel's

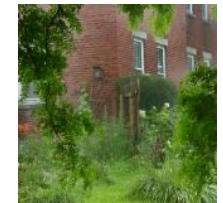

(b) He's

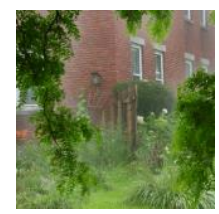

(e) Zhu's

Group II-House

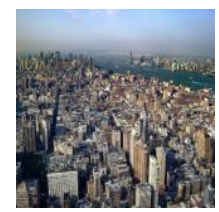

(b) He's

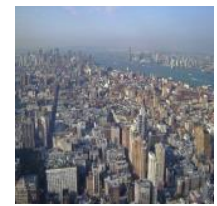

(e) Zhu's

Group III-City

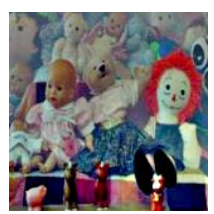

(c) Retinex's

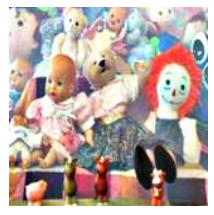

(f) Our

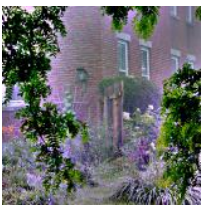

(c) Retinex's

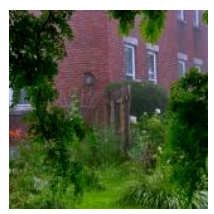

(f) Our

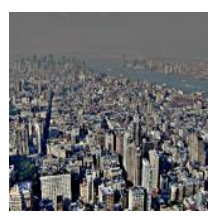

(c) Retinex's

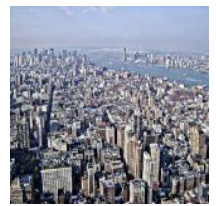

(f) Our method

\section{FIGURE III. DIFFERENT ALGORITHMS' DEFOGGING RESULTS}

Besides, we also use the visible edge gradient ratio, information entropy, statistical feature parameters and details before and after the image restoration as the objective evaluation index of the dehazing effect.
T ABLE I. DIFFERENT ALGORITHMS' TIME CONSUMING

\begin{tabular}{cccc}
\hline Image & Doll(s) & House(s) & City(s) \\
Proposed & $\mathbf{1 . 4 0 5}$ & $\mathbf{4 . 1 6 6}$ & $\mathbf{1 3 . 7 4 2}$ \\
He's & 9.863 & 73.572 & 113.68 \\
Retinex's & 4.789 & 4.953 & 15.016 \\
Tarel's & 4.144 & 24.677 & 92.393 \\
Zhu's & 1.422 & 4.241 & 14.482 \\
\hline
\end{tabular}

As can be seen from Table 2, Zhu algorithm obtains a higher $X$, but a smaller $\bar{r}$ and a larger $S$; Tarel algorithm has a larger $\bar{r}$, but its $S$ is too large; Retinex algorithm obtains a larger $\bar{r}$, but its corresponding $H$ is too small. In contrast, both $\mathrm{He}$ algorithm and the proposed algorithm in this paper appear to be more balanced in all aspects, but most of the algorithms above have higher $\bar{r}$ and $H$ than Zhu algorithm, while have higher $X$ and lower $S$, as is consistent with the subjective comparis on results.

T ABLE II. PERFORMANCE COMPARISON

\begin{tabular}{llllll}
\hline images & Method & $H$ & $\bar{r}$ & $S$ & $X$ \\
\hline \multirow{4}{*}{ Doll } & Original & 14.68 & 12.96 & 1 & 0.19 \\
& He's & 14.92 & 22.85 & 0.67 & 0.22 \\
& Retinex's & 14.59 & 29.32 & 0.60 & 0.33 \\
& Tarel's & 15.09 & 26.13 & 0.76 & 0.41 \\
& Zhu's & 14.89 & 19.14 & 0.79 & 0.21 \\
& Our & 15.09 & 39.15 & 0.46 & 0.23 \\
\hline \multirow{4}{*}{ City } & Original & 15.19 & 26.15 & 1 & 0.26 \\
& He's & 16.78 & 40.86 & 0.67 & 0.22 \\
& Retinex's & 15.75 & 49.47 & 0.28 & 0.18 \\
& Tarel's & 18.14 & 40.49 & 0.50 & 0.26 \\
& Zhu's & 16.28 & 29.61 & 0.83 & 0.26 \\
& Our & 18.14 & 60.65 & 0.71 & 0.27 \\
\hline \multirow{6}{*}{ House } & Original & 15.46 & 26.11 & 1 & 0.34 \\
& He's & 16.59 & 29.86 & 0.66 & 0.37 \\
& Retinex's & 15.33 & 57.29 & 0.45 & 0.14 \\
& Tarel's & 16.83 & 33.37 & 0.61 & 0.37 \\
& Zhu's & 16.32 & 28.11 & 0.81 & 0.35 \\
& Our & 16.83 & 31.05 & 0.63 & 0.43 \\
\hline \multirow{6}{*}{} & & & & &
\end{tabular}

VI. CONCLUSION

In this paper, we propose a fast and efficient foggy image sharpening algorithm based on depth of field estimation. The algorith $\mathrm{m}$ can effectively solve color attenuation problem and incomplete defogging of the existing mainstream algorithms. Our method not only avoids the calculation of the transmission estimation but also improves the real-time performance. Besides, to some extent, our method overcomes Zhu algorithm's insufficiency for defogging and its capability for defogging and universality are better.

\section{ACKNOWLEDGMENT}

This work was supported by the Fundamental Research Funds for the Central Universities in China under Grant No 
2013 B32514 and the National Natural Science Foundation of China under Grant No 61563036.

\section{REFERENCE}

[1] Gonzalez R C, Woods R E. Digital Image Processing. Reading, MA: Addison-Wesley, 1992.

[2] Yu J, Li D, Liao Q. Color constancy-based: visibility enhancement of color images in low-light conditions. Acta Automatica Sinica, 2011, 37(8): 923-931.

[3] John J, Wilscy M. Enhancement of weather degraded video sequences using wavelet fusion. Proceedings of the 7th IEEE International Conference on Cybernetic Intelligent Systems. London: IEEE, 2008: 1-6.

[4] He K, Sun J, Tang X. Single image haze removal using dark channel prior. Computer Vision and Pattern Recognition, 2009. CVPR 2009. IEEE Conference on. IEEE, pp:1956-1963.

[5] Zhu Q, Mai J, Shao L. A fast single image haze removal algorithm using color attenuation prior. IEEE Transactions on Image Processing A Publication of the IEEE Signal Processing Society, 2015, 24(11): 3522-3533.

[6] Fattal R. Single image dehazing. ACM Transactions on Graphics, 2008, 27(3): 1-9.

[7] Cheng F, Cheng C, Lin P, et al. A hierarchical airlight estimation method for image fog removal. Engineering Applications of Artificial Intelligence, 2015, 43:27-34.

[8] Yan S J. Research and improvement of white balance processing algorithm for digital image [D]. Shanghai Jiaot ong University, Shanghai, China, 2007.

[9] Gu Z, Ju M, Zhang D. A single image dehazing method using average saturation prior. Mathematical Problems in Engineering, 2017:1-17.

[10] Ju M, Zhang D, Wang X. Single image dehazing via an improved at mospheric scattering model. Visual Computer, 2017, 33(12):1-13.

[11] Tarel J P, Hautiere N, Caraffa L, et al. Vision enhancement in homogeneous and heterogeneous fog. Intelligent Transportation Systems Magazine IEEE, 2012, 4(2):6-20.

[12] Retinex algorithm [M]. Springer US, 2014. 